
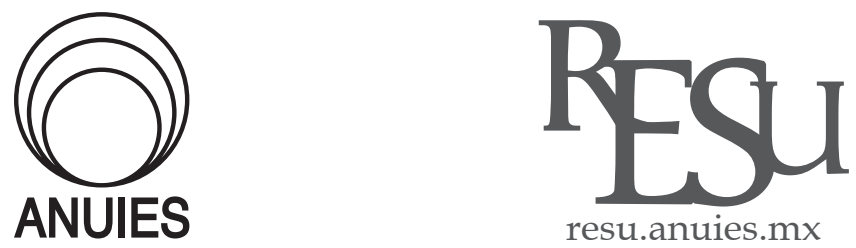

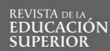

ARTí́CULO

\title{
El Tecnológico Nacional de México. Emergencia y procedencia
}

Tecnológico Nacional de México. Emergency and source

Reinalda Soriano Peña*

*Tecnológico Nacional de México, sede Centro Interdisciplinario de Investigación y Docencia en Educación Técnica Correo electrónico: reynaspea@yahoo.com.mx

Recibido el 29 de mayo del 2019; aceptado el 15 de noviembre del 2019

\section{Resumen}

El artículo presenta resultados parciales de una investigación que aborda la constitución de sujetos educativos desde el análisis de las políticas educativas. ¿Cuál es la emergencia y la procedencia del Tecnológico Nacional de México que forma principalmente a ingenieros? Se exponen los referentes conceptuales y metodológicos de la investigación: el análisis político de discurso (Laclau y Mouffe, 1987), desde el ámbito educativo en México a las contribuciones de Buenfil (1994, 2011, 2018); y los conceptos procedencia y emergencia de Foucault (1992). Se analiza la emergencia de los primeros Institutos Tecnológicos y la emergencia y la procedencia del Tecnológico Nacional de México (теслм). Finalmente, expone algunas conclusiones.

Palabras clave: Formación, ingeniería, educación superior, política educativa, identidad 


\begin{abstract}
The article presents partial results of a research that addresses the constitution of educational subjects after the analysis of educational policies. What was the beginning and descent of the Tecnológico Nacional de México (TecNM). that mainly trains engineers? The conceptual and methodological research referents are exposed: the political analysis of discourse (Laclau and Mouffe, 1987), from the educational sphere in Mexico, the contributions of Buenfil (1994, 2011, 2018); and the concepts of descent and emergence of Foucault (1992). The emergence of the first Technological Institutes and the emergence and descent of the TecNM are analyzed. Finally, some conclusions are exposed.
\end{abstract}

Keywords: Training, engineering, higher education, educational policy, identity

\title{
Introducción
}

$\mathrm{L}$ a formación de ingenieros es un campo de conocimiento poco explo- rado, principalmente desde una mirada pedagógica. El artículo se deriva de una investigación (cualitativa, documental e interpretativa), en proceso, cuyo objeto de estudio es la constitución de sujetos educativos. Este artículo pretende dar respuesta a la pregunta ¿Cuál es la emergencia y la procedencia del Tecnológico Nacional de México (тесNм), institución de educación superior pública que forma principalmente a ingenieros?

El propósito del trabajo es dar cuenta de cómo ha llegado a ser lo que es hoy el TecNM, cuáles son las huellas y marcas de las políticas educativas implementadas, en dónde se impulsa un sentido de ser ingeniero, la formación profesional y qué proyecto de país se está impulsando. ¿Cuáles son esos modelos identitarios que se proponen para la formación de ingenieros en el тесNM?

No obstante, no puedo dejar de reconocer que las propuestas identitarias se construyen en diferentes espacios y configuraciones discursivas donde se lleva a cabo la formación de ingenieros: I) los programas y documentos "oficiales", por medio de las políticas educativas; iI) las prácticas educativas en torno a la formación de ingenieros; III) las imágenes del ser ingeniero que se construyen por parte de la institución y que son presentadas como socialmente aceptables.

En este artículo expongo únicamente las políticas educativas que crearon las condiciones para la procedencia y la emergencia del TесNM. Las políticas educativas no son entendidas aquí sólo como enunciados que planean y or- 
ganizan, sino que también operan a través de estas políticas como efectos de poder. Al respecto Foucault (2008) considera que son tecnologías que disciplinan y contribuyen a la apropiación de ciertos modos de ser, de comportarse, de actuar.

Eso permite comprender que la emergencia de los primeros Institutos Tecnológicos (ITS) no se da de manera aislada, es producto de los antagonismos y polarizaciones políticas de los años treinta, cuarenta y cincuenta en nuestro país, al darse una disputa de proyecto de país, por un lado por el cardenismo, y por el otro, con el avilacamachista y el alemanista. Es en este contexto como se crean los primeros IT.

El escrito expone en primer lugar los referentes conceptuales y metodológicos de la investigación: I) como perspectiva general el análisis político de discurso (Laclau y Mouffe, 1987), desde el ámbito educativo en México con las contribuciones de Buenfil (1994, 2011, 2018); II) se recuperan los conceptos procedencia y emergencia de Foucault (1992) para llevar a cabo un ejercicio genealógico. En segundo lugar, el referente empírico que alude a la emergencia de los primeros Institutos Tecnológicos, considerado el momento fundador de un archipiélago tecnológico ${ }^{1}$; en tercero, la emergencia y la procedencia del TecNM. Finalmente, se presentan algunas conclusiones.

\section{Referentes conceptuales y metodológicos de la investigación}

La dimensión conceptual es necesaria en todo proceso de investigación. Una perspectiva que me aporta al trabajo investigativo es el análisis político de discurso (Laclau y Mouffe, 1987), y en México, en lo educativo, las contribuciones de Buenfil (1994, 2011, 2018), pues permiten recuperar herramientas conceptuales y analíticas que apoyan a la comprensión de la constitución de sujetos educativos y la formación de identidades sociales, como son las de los IT.

Por un lado, Laclau y Mouffe (1987), a partir de los aportes gramscianos, incorporan una lógica de lo político como constitutivo de lo social, y uno de ellos es que toda práctica política constituye una práctica educativa. Desde un pensamiento postmarxista, se reconoce la dimensión educativa en la acción política, ya que ambas son inseparables en las prácticas que se examinan (Buenfil, 2018). Desde este posicionamiento es relevante la distinción y el

\footnotetext{
${ }^{1}$ Esto formará como categoría intermedia que expondré en páginas más adelante.
} 
análisis de la relación entre lo político, la política, las políticas ${ }^{2}$ en lo educativo. Por otro lado, este horizonte teórico abre la posibilidad de comprender y analizar la constitución de los sujetos en los procesos educativos. Es en las prácticas educativas, como prácticas discursivas, ${ }^{3}$ en donde se llevan a cabo diversas modalidades de significatividad.

Lo educativo alude [...] a un registro, no a una región de hechos, instituciones, contenidos o estrategias de enseñanza. Concierne a la formación del sujeto, a lo que está implicado en su constitución, a lo que permite distinguir aquello que marca un antes y un después, y en este sentido alude a su dimensión de acontecimiento ${ }^{4}$ (Buenfil, 2018: 259).

Estos planteamientos, considero, permiten articular una mirada pedagógica en relación con las políticas educativas en donde se construyen modelos identititarios dirigidos a los diferentes sujetos en proceso de formación. Desde las políticas educativas se ofrecen modelos de identificación que interpelan a los actores principales, docentes y estudiantes en proceso de formación como ingenieros en el TесNM, e inciden en el sentido de ser ingeniero que se pretende impulsar y que se espera desarrollen en su actuar profesional y en su vida cotidiana.

Me interesa señalar que pueden observarse diferencias entre las propuestas identitarias que se construyen en los espacios y configuraciones discursivas donde se lleva a cabo la formación de ingenieros: programas y documentos "oficiales", políticas educativas; prácticas educativas en torno a la formación de ingenieros; las imágenes del ser ingeniero que se construyen desde la institución y que son presentadas como socialmente aceptables, etc. Sin embargo, estas distinciones no implican que los tres espacios estén separados o se opongan, ya que tanto las prácticas educativas como las imágenes identitarias que se despliegan en las instituciones están impregnadas (no determinadas) por las políticas educativas y otras formaciones culturales del contexto (como se observará en el análisis del referente empírico más adelante).

\footnotetext{
${ }^{2}$ Lo político es constitutivo de lo social (Laclau y Mouffe, 1987). La política es en donde se definen las fronteras entre lo que se considera o no válido para los sujetos, es la arena de combates y acuerdos parciales y temporales (Buenfil, 2011). Y las políticas son los dispositivos gubernamentales, que siempre son sujeto de resignificación (Buenfil, 2011).

${ }^{3}$ Discurso entendido como configuración de significados, que incluye prácticas lingüísticas y extralingüísticas. Se refiere a la construcción de significaciones por los sujetos sociales, de manera individual o colectiva.

${ }^{4}$ Acontecimiento tiene que ver con las dimensiones ontológica y epistemológica, para dar cuenta de la transformación subjetiva del sujeto educativo (el antes y el después) de un llamado o propuesta identitaria (Buenfil, 2018).
} 
Los conceptos de procedencia y emergencia (Foucault, 1992) desde una perspectiva de investigación genealógica, no pretenden la búsqueda del origen de algo, sino analizar los diversos acontecimientos, eventos, contingencias, situaciones que permiten comprender la procedencia y que generalmente se presentan como dispersión o como eventos disgregados o sin ninguna articulación. La emergencia posibilita dar cuenta del surgimiento en el marco de un estado de fuerzas en pugna. Desde esta perspectiva, no pretendo presentar la verdad absoluta del origen de los ITs y del TесNM, sino más bien alumbrar situaciones, posiciones o momentos que posibilitaron su emergencia y procedencia.

La presente investigación es cualitativa, documental, de campo e interpretativa. La propuesta metodológica de la investigación más amplia que se construyó es a partir de unidades analíticas ${ }^{5}$. La que aquí se presenta se conforma con las producciones discursivas de: I) Protagonistas clave del TecNM: la información que se obtuvo fue mediante entrevistas semi- abiertas, para lo cual se confeccionó un guión de entrevistas y la incorporación de otras preguntas no contempladas inicialmente; las entrevistas se realizaron en el mes de septiembre de 2018 y las transcripciones durante noviembre a diciembre de 2018. II) Documentos oficiales en relación con las políticas educativas, principalmente en relación con la creación de los primeros tecnológicos y con el Decreto de creación del actual TecNM. III) La consulta de bibliografía en relación con la creación de los primeros tecnológicos; la indagación documental se llevó a cabo durante los meses de agosto de 2018 a marzo de 2019.

Un primer análisis de las entrevistas y de la revisión documental posibilitó construir un conjunto de ejes analíticos (emergencia y procedencia del TecNM; formación y formación profesional; políticas educativas e identidad). La sistematización de la narrativa de los actores clave del TecNM y de los documentos oficiales en un cuadro general, posibilitó integrar fragmentos con base en un eje analítico. Este trabajo permitió ir definiendo diversas categorías de análisis (archipiélago tecnológico; ser ingeniero; identidad profesional; regionalización). El resultado del concentrado fue contar con un conjunto de producciones discursivas relacionadas con distintos aspectos de la política educativa del TесNм y sus propuestas de ser ingeniero que permiten comprender la emergencia y la procedencia de esta institución educativa. Del conjunto de la información en este artículo se presenta lo relativo a: I) el

\footnotetext{
${ }^{5}$ Por ejemplo: la producción de las políticas educativas producto de las reformas, su circulación, su diseminación y sus apropiaciones. Las unidades de análisis permiten conformar los núcleos de significado que pueden ser objeto de estudio para proceder a su clasificación y su recuento.
} 
contexto y el momento fundadores de los IT; y II) el contexto y la creación del TесNM, que permiten identificar algunas huellas y desplazamientos en la política educativa que se ha construido para la formación de ingenieros. Interesa el sentido de ser ingeniero que subyace en políticas, a partir de las cuales se construyen modelos de identificación.

A continuación se presenta la emergencia de los primeros ITs y el contexto para comprender las condiciones que posibilitaron el inicio de la conformación de un archipiélago tecnológico y comprender cómo ha llegado a ser lo que es hoy el тесNм, en términos de su emergencia y su procedencia, un ejercicio genealógico para analizar la singularidad de los sucesos (Foucault, 1992).

\section{El momento fundador del archipiélago tecnológico. Los primeros institutos tecnológicos}

La noción de archipiélago ha sido útil como metáfora para la discusión del pensamiento filosófico y político, para dar cuenta de la dispersión, la heterogeneidad y la multiplicidad de configuraciones discursivas (Ruiz, 2001). ${ }^{6} \mathrm{La}$ noción de archipiélago tecnológico, como categoría intermedia me posibilita explicar y dar cuenta de los múltiples espacios de formación de los ingenieros en los diferentes Iт federales y descentralizados que se encuentran en México. Si bien forman parte del TесNM, muestran particularidades de acuerdo con las regiones del país en que se encuentran. Aunque a simple vista parecen inconexos, no están separados ni son independientes, tienen una estabilidad relativa pero no son fijos, sino que están en permanentes construcción y reconstrucción, y entre ellos se van desplazando sentidos políticos y educativos de ser ingenieros que se ofrecen por medio de las políticas y los programas educativos.

Para mostrar algunos aspectos del archipiélago tecnológico y cómo está conformado el тесNм, recurro a algunos antecedentes históricos de cómo es que se crearon los primeros IT de Chihuahua y Durango durante la década de los años cuarenta en México. Su importancia radica en que constituyen el momento fundador (Fernández, 1994) de un archipiélago tecnológico en el

\footnotetext{
${ }^{6}$ Mercedes Ruiz (2001) ha construido la categoría intermedia archipiélago educativo para su investigación doctoral, que resultó inspiradora. Esta categoría permite reconocer que la identidad se configura en la diversidad de espacios educativos y políticos, que no son independientes, sino que presentan conexiones por las formaciones tectónicas profundas y a través de microfisuras que permiten conectar un espacio con otro, como lo señala la autora.
} 
cual se busca normar y regular una manera distinta de formar ingenieros. Son reconocidos actualmente como los "padres" del sistema tecnológico que al inicio dependieron del IPN, posteriormente de la SEP, y hoy compiten con el IPN, la UNAM y otras instituciones de educación superior pública en donde se forman también ingenieros.

La emergencia de los primeros IT (Chihuahua y Durango), a fines de la década de los años cuarenta de fines del siglo pasado, puede explicarse por las condiciones no sólo a nivel nacional sino también internacional, que posibilitaron cambios en la educación superior.

En el ámbito internacional estas condiciones se caracterizan por el desarrollo de la Segunda Guerra Mundial (1939-1945) que impacta a varios países, tanto política y económica como socialmente; la creación de la Organización de las Naciones Unidas para la Educación, la Ciencia y la Cultura (UnESCO) (1945). La Primera Conferencia General de la UnESCo fue en París (1946) y la Segunda Conferencia en México (1947), y se propuso en esta última un mínimo de educación de base para desempeñarse en un mundo moderno y una educación técnica; los avances en el campo de la ciencia y de la tecnología; el impulso creciente de una economía de mercado a nivel mundial; y el capitalismo y la industrialización pujante a nivel internacional.

En el ámbito nacional interesa destacar la incipiente industrialización iniciada en los años treinta con el cardenismo; cobra mayor impulso con el modelo de sustitución de importaciones a partir de la incipiente oferta de productos debido a la Segunda Guerra Mundial. En este contexto asume la presidencia Manuel Ávila Camacho (1940-1946) e impulsa una política de industrialización; y se continúa con esta misma política durante la posguerra durante la presidencia de Miguel Alemán (1946-1952). La época de la posguerra incidió para crear condiciones para la transformación de la educación superior en el país (hasta 1950 existían alrededor de 12 instituciones de educación superior pública, entre las cuales estaban la UNAM y el IPN). Durante los años setenta se funda la Universidad Autónoma Metropolitana, se lleva a cabo la desconcentración de la UNAM y se crean las entonces Escuelas Nacionales de Educación Superior (Zaragoza, Iztacala, Acatlán y Aragón), por lo que después del movimiento estudiantil de 1968 hay una ampliación de la oferta educativa de la educación superior pública.

Se puede considerar que tiene lugar un proceso de consolidación de las instituciones que se crearon desde los años cincuenta, además de la apertura de institutos tecnológicos y apoyo a las instituciones privadas. ${ }^{7}$ Se intensifi-

\footnotetext{
${ }^{7}$ Desde 1943 se habían fundado instituciones privadas donde se impartía también enseñanza tecnológica (Universidad Iberoamericana y el Instituto Tecnológico de
} 
can los procesos de modernización económica (basados en la economía de mercado y el uso de tecnologías industriales), así como la dependencia económica, académica y política de países del exterior (principalmente de Estados Unidos), y un crecimiento demográfico y de urbanización de las grandes ciudades.

Este contexto permite analizar los procesos históricos pero no desde una historia unificada, con un sentido único y como evolución. Es desde este posicionamiento que pretendo dar cuenta de un contexto problematizador de donde proceden y emergen las políticas educativas que pretenden no sólo planear y organizar, sino que también tienen efectos de poder. Son tecnologías que disciplinan y contribuyen a la apropiación de ciertos modos de ser, de comportarse, de actuar (Foucault, 2008).

Considero que la noción de procedencia me apoya para dar cuenta de las marcas singulares que subyacen en las políticas en relación con la formación y los sentidos de ser ingeniero. Cómo, a través de la historia, estas propuestas se han mantenido o se han difuminado de acuerdo con el contexto histórico y el proyecto de país.

Cabe reconocer los momentos contingentes que se presentaron alrededor de las disputas y de la política educativa del momento. Es por ello que interesa colocar la mirada en la emergencia de acontecimientos y las diversas fuerzas políticas (Gobierno Federal, autoridades, etc.) que logran o no articularse para la construcción o impulsar proyectos para la formación de ingenieros.

Es desde las políticas educativas como se pretende impulsar un proyecto de país en pugna con el diseñado durante el gobierno de Lázaro Cárdenas del Río (1934-1940); que son desprestigiadas y desmanteladas por las reformas de Ávila Camacho (1940-1946) y Miguel Alemán Valdés (1946-1952). Una de las características del modelo educativo de la educación superior cardenista es el sentido que se otorga a la enseñanza, como un medio para lograr la independencia tecnológica para el servicio del pueblo. Es de destacar que se abre el espacio para un proyecto educativo acorde con el proceso de industrialización enfatizando en la educación técnica, y se promueve con ello el colectivismo y el desarrollo de la tecnología (Buenfil, 2004). Claros indicios de esta meta pueden observarse en diversas intervenciones durante su gira presidencial. $^{8}$

Estudios Superiores de Monterrey - ITESM-).

${ }^{8}$ Existe una compilación publicada en 1934, La jira [sic] del General Lázaro Cárdenas. Síntesis ideológica México, Secretaría de prensa y propaganda del C. E. N. del P. N. R. 
Cada hijo de obrero que penetra en las escuelas de instrucción superior, universitarias o técnicas, es un líder en potencia que pierden el sindicato o la organización campesina, y un técnico más que irá a rendirse al servicio de los poseedores de la riqueza. La crema del proletariado pasa a ser, por virtud de este fenómeno, rico manjar al gusto de la burquesía (Townsend, 1959, en Buenfil, 1994: 168).

La propuesta consistía en que la enseñanza técnica apoyase a la industria, pero nacional. Ante la negativa de la UNAM de participar en el proyecto de país cardenista, se da una disputa y es así como como emerge el IPN en 1937 como una institución de educación superior pública alternativa a la UNAM claramente liberal. En el IPN se logra una organización institucional y unificada de diferentes escuelas formadoras de ingenieros (Escuela de Ingeniería Mecánica y Eléctrica, y la Escuela Superior de Construcción) buscando precisamente atender los intereses y necesidades del país y formar a los ingenieros que respondieran a dichas necesidades con independencia del extranjero. En ese entonces cien mil extranjeros controlaban el 99\% de las industrias extractivas y un $60 \%$ de las de transformación (Guevara Niebla, 1983).

En las décadas de los cuarenta y cincuenta del siglo xx, se lleva a cabo el desmantelamiento de la educación cardenista. Durante el gobierno presidencial de Manuel Ávila Camacho (1940-1946) se impulsa un proyecto educativo centrado en la competencia y las especializaciones orientadas a las industrias urbanas. Las escuelas fueron entendidas ya no como forma de reducir la desigualdad sino como un medio para ascender en la escala social (Buenfil, 2004). Las esperanzas estaban cifradas en la industrialización del país, pero con una fuerte dependencia económica y tecnológica.

Cabe destacar que el gobierno alemanista del país se centró también en la política de industrialización del país, dando continuidad al proyecto avilacamachista. Con la finalidad de que la economía nacional dejara de depender de la producción agrícola, en educación superior también se consideró necesario ampliar también la enseñanza tecnológica pública. Una característica de este periodo gubernamental fue la creciente dependencia económica y tecnológica principalmente ante Estados Unidos, y México ocupó el papel de productor de materias primas y de importador de productos manufacturados. La intención era propiciar desde el gobierno la llegada al país de inversiones extranjeras, particularmente de Estados Unidos.

Es así como se puede comprender el por qué se consideró que en Chihuahua, por su cercanía con el país más poderoso del mundo y con un mercado industrial, emergiera en 1948 un Instituto Politécnico (Sáenz, 1999), unos meses después de la devaluación económica que tuvo el país en julio de 1948, primero la apertura de los it de Chihuahua, por decisión de Miguel Alemán 
Valdés el 21 de enero de 1948, y posteriormente, en el mismo año, el IT de Durango.

La creación del ITch tuvo como objetivo "[...] forjar individuos con capacidad suficiente, técnica y moral, que les permita dedicarse a la explotación de las riquezas del estado, en forma racional, para bien de sus habitantes, así como para el resto del país" (Sáenz, 1999: 21). Se presentó su apertura como algo nuevo que no tenía antecedentes en México, es por ello que para algunos autores se consideró como el primero en su tipo en México, "[...] no existía punto de referencia o un modelo a seguir y los estudios debieron partir de cero" (Sáenz, 1999: 21). La paradoja es que la mayoría de los docentes de ese IT eran egresados del IPN, con lo que indicios de la procedencia politécnica son insoslayables.

Cabe destacar la participación de autoridades de la Secretaría de Educación del gobierno federal y del IPN como responsables realizar un estudio para definir qué carreras ofrecer de acuerdo a la infraestructura que se requería y las necesidades del estado de Chihuahua. ${ }^{9}$ Es así como se decide que la carrera a impartir por ese IT es la de Ingeniero Industrial, con especialidad de productos vegetales, animales o minerales. Los cursos iniciaron el 19 de septiembre de 1949, pero autores como Galindo $(2010)^{10}$ afirman que fue el 2 de septiembre de ese mismo año, asimismo, que el IT de Durango inició actividades el 2 de agosto de 1948.

De manera que el segundo en la república del sistema de tecnológicos fue el de Chihuahua, aunque fue el primero en ofrecer carreras profesionales y sus respectivos directores fundadores fueron José Gutiérrez Osornio [...] y Gustavo Alvarado Pier [...] ambos egresados de la ESIME del Instituto Politécnico Nacional (Galindo, 2010: 1; el énfasis es mío).

Es así que se considera que el ITch se convierte en el primer tecnológico, al crearse el 21 de enero de 1948 e impartir la carrera de ingeniería industrial, y en 1954 egresa la primera generación, lo que posibilitó que también se ofrecieran niveles de vocacional, subprofesional y capacitación técnica para los trabajadores (Sáenz, 1999).

\footnotetext{
${ }^{9}$ El Secretario de Educación, Manuel Gual Vidal, designó a los ingenieros Gustavo Alvarado Pier (exdirector del IPN y Carlos M. Tello (Jefe del departamento de Capacitación Técnica para Trabajadores del IPN) como responsables de realizar el estudio, apoyados por el ingeniero Alejandro Guillot (recién nombrado en ese entonces como director del IPN) (Sáenz, 1999).

${ }^{10}$ Carlos Galindo Cisneros es egresado del гтсн, de la carrera de Ingeniería Mecánica en 1962 y ha sido docente en los iт de Ciudad Juárez, Hermosillo, Puebla, Ciudad Juárez.
} 
También interesa resaltar el comunicado del primer director del ITch durante la ceremonia de inauguración de clases el 19 de septiembre de $1949^{11}$ en el Palacio de Gobierno de Chihuahua, que entre otras cosas destacó el papel de la educación técnica para el país:

[...] El establecer en puntos estratégicos de nuestro país Institutos Tecnológicos, es una obra patriótica [...]. La semilla que hoy se siembre es un campo fértil como lo es la provincia, donde las riquezas naturales aún no han sido intensamente explotadas, está esperando la mano del hombre y la técnica que las industrialice para convertirlas en fuente de bienestar y de progreso para sus habitantes. [El ITCh] no tiene solo una importancia local, está ayudando a resolver un problema nacional, es el laboratorio de experimentación de nuevos métodos y nuevas técnicas, es la valiosa contribución de Chihuahua al perfeccionamiento de la enseñanza técnica [...] (Ing. Gustavo Alvarado Pier en Sáenz, 1999: 28) (El énfasis es mío).

Cabe destacar que el Estado era el principal empleador de los ingenieros, en empresas paraestatales y dependencias gubernamentales, por lo cual ellos alcanzaron una mayor participación en el poder público y económico, lo que posibilitó un ascenso social y profesional de la ingeniería mexicana. Esto creó las condiciones para que esta profesión alcanzara reconocimiento social y legitimación profesional en los años cuarenta e inicios de los cincuenta del siglo $x x$, por lo que se consolida como profesión al fortalecerse la profesionalización de los ingenieros (Ruiz, 2008). ${ }^{12}$

El proyecto de formación consistía en que los futuros ingenieros "se convirtieran" en agentes tecnológicos (Ruiz, 2008) para contribuir a la modernización del país, organizar social y académicamente esta profesión; otorgar una visión a la profesión y su enseñanza; y construir una identidad profesional, de prestigio y de estatus social:

[...] la ingeniería se consolida como una de las profesiones más importantes para el país [...] en la conformación de las élites industriales [...] en el surgimiento de las primera asociaciones de ingenieros [...] con mucha influencia en las decisiones de carácter tecnológico y la formación de pro-

\footnotetext{
${ }^{11}$ Las clases comenzaron el 20 de septiembre de 1949 en un horario de 9:00 a 13:00 horas y de 15:00 a 17:00 horas. La primera generación que se inscribió para estudiar ingeniería industrial estuvo conformada por 20 estudiantes. Es hasta el 22 de octubre de 1952 cuando se inauguraron las instalaciones del iтch por el expresidente Alemán Valdés.

${ }^{12}$ Algo muy similar a Estados Unidos, en donde los ingenieros participaban como actores clave en el ámbito institucional: en el gobierno, las empresas y las escuelas de ingeniería (Tang, 2000 en Ruiz, 2008).
} 
fesionales [...] afiliaba a los más destacados ingenieros mexicanos [ocupaban] cargos superiores del gobierno o en la academia (Ruiz, 2008: 228).

Es de mi interés resaltar cómo fue el crecimiento exponencial del archipiélago tecnológico después de su momento fundador, en un contexto tan diverso en donde se construyen diversas políticas. Por ejemplo, este auge de la ingeniería en el país en el que emergen los primeros IT's (Chihuahua y Durango en 1948 y Tamaulipas en 1954), tuvo quiebres importantes durante los años sesenta. La expansión industrial y de servicios intensificó una diversificación en los espacios productivos, se da un aumento de puestos técnicos y administrativos principalmente, surgen nuevos de campos de ocupación pero asociados a los servicios de las zonas urbanas, y una intensa división del trabajo que incide en la polarización de puestos jerárquicos.

En los setenta se implementa la política educativa "de apertura democrática" para este nivel educativo, en donde se amplían las oportunidades de ingreso a la educación superior, es así como el archipiélago tecnológico de los ITs crece de manera vertiginosa. De 1978 a 1988 se fundan 12 nuevos Institutos Tecnológicos, por lo que se eleva la cifra a 70.

La política educativa en las décadas de los años setenta y ochenta hace posible que la educación técnica reciba un gran impulso al crearse un sistema de educación denominado "tecnológico" a escala nacional, que queda bajo control de la SEP (Weiss y Bernal, 1982). Durante el periodo 1970-1980 la matrícula escolar en la educación superior fue de las más altas, pero también emerge desde años anteriores un problema importante que es la permanencia restringida (reprobación, deserción, abandono, titulación). No todos los estudiantes contaban con perfiles socioeconómicos y de capital cultural que les posibilitara mantenerse en la educación superior. Este problema tampoco fue ajeno a los ITs.

Al respecto, Fernández (1994), plantea que una institución es en principio un objeto cultural que expresa cierta cuota de poder social. Es por ello que puede entenderse que para atender el problema de la permanencia restringida, se considerara impulsar y desarrollar instituciones y modelos alternativos de enseñanza.

La SEP cambia su concepción de desarrollo frente a la de años anteriores. La promesa que se impulsaba por medio de la política educativa desarrollista era que la educación era un bien social y todos podían tener acceso a ello y era la vía de movilización social para mejorar las condiciones de vida a la población. Es a fines de los setenta e inicios de los ochenta, con la crisis económica que hubo en el país, que se cuestionan dichas promesas. Durante los años noventa del siglo xx la educación superior conforma un sistema de 
instituciones tan diverso, con estructuras de organización administrativa y académica tan desigual, que no redujo el problema de la deserción y el rezago educativo.

Para afrontar los problemas de la educación superior a fines del xx e inicios del siglo xxI, se modifica la política y emergen los tecnológicos descentralizados de los gobiernos estatales (fines de los años 90). La nominación que se les da es para diferenciarlos de los ITs federales, y son nombrados como Tecnológicos de Estudios Superiores, por lo que el archipiélago crece también de manera significativa y emerge un personal académico más joven, pero con diferentes condiciones laborales menos estables.

Los ITs se establecen divididos en regiones que se extienden geográficamente a lo largo y ancho del país. La regionalización tecnológica también da cuenta de la creación de un centro, de la periferia, de lo local, y aunque pareciera sólo una distribución física, también influye en los significados que construyen los actores en los diferentes tecnológicos. No es lo mismo ser académico o estudiante de un tecnológico del norte, del sur o del centro del país (como puede observarse en las entrevistas).

En este contexto funda, en el siglo xxI, el Tecnológico Nacional de México (тесNM) en 2014, a partir del Decreto de creación durante el periodo presidencial de Enrique Peña Nieto, en el marco de una Reforma Educativa derivada de la política educativa.

\section{La procedencia y la emergencia del TecNM}

En primer lugar presento algunos datos del diagnóstico (1996-2006) realizado a instancias de la SEP para ser presentado en la Organización para la Cooperación y el Desarrollo Económicos (OCDE). En la primera década del siglo XXI existe un sistema de educación superior muy complejo, tanto en su tamaño como en su heterogeneidad, con una elevada matrícula (Rubio, 2006). Es un reto brindar un servicio educativo que atienda a la política educativa que se ha caracterizado en las últimas décadas del siglo pasado y las primeras del XXI, en la búsqueda de "cobertura, calidad, pertinencia, diversificación, federalismo descentralizados, equidad, gestión, planeación, evaluación y coordinación" (Tamez, 2006: 12) ${ }^{13}$ El sistema de educación superior (periodo 1996-2006), siguiendo a este autor, estaba conformado por nueve subsiste-

\footnotetext{
${ }^{13}$ Reyes S. Tamez Guerra, Secretario de Educación Pública, en el gobierno de Vicente Fox (2000-2006).
} 
mas, entre ellos el de tecnológicos públicos (federales y descentralizados) ${ }^{14}$ que posteriormente se concentraron en el hoy TесNM).

Este diagnóstico realizado durante el gobierno de Vicente Fox es muestra de la política educativa en su momento; el ideal de plenitud estaba centrado en la cobertura, la calidad, la pertinencia, la diversificación, el federalismo descentralizado y la equidad.

Este es el contexto de emergencia del тесNM, desde las instancias oficiales se plantea que la intención de crearlo es para integrar los ITs en una sola institución. Pareciera que el interés es más administrativo que educativo, pues su finalidad era que fuese líder en la formación de ingenieros en México (Gamino, Acosta y Pulido, 2016). En esta apertura ${ }^{15}$ operó una lógica administrativa políticamente vistosa y una configuración discursiva que incluye la creación de instituciones, su sentido, y su financiamiento, todo en consonancia con el Plan Nacional de Desarrollo 2013-2018 de la administración de Peña Nieto.

Desde ese entonces ha tenido diversos procesos de transformación con la puesta en marcha de un proyecto de transformación institucional, entre ellos la modificación o revisión del modelo educativo, el cambio de administración, gestión y operación institucionales. Con ello ha buscado dar respuestas a los problemas en la vida del sistema tecnológico en esta institución educativa.

¿Cuáles son las condiciones que exigen un cambio en la institución? Principalmente se relacionan con el ámbito internacional (con los procesos asociados con la globalización, el desarrollo de economías informacionales; el vertiginoso cambio de las tecnologías de información y comunicación) y que a través de organismos internacionales impactan en las políticas educativas de las instituciones de educación superior a nivel mundial, que exigen nuevos modelos y propuestas curriculares, la producción de nuevos conocimientos, y nuevas maneras de trabajo académico en las instituciones educativas.

En el ámbito nacional son diversos los aspectos, sobresale la lucha por el financiamiento otorgado a la educación superior, la construcción de propuestas para obtener apoyo y reconocimiento del trabajo desarrollado en las insti-

\footnotetext{
${ }^{14}$ En el periodo estaba conformado por 211 institutos, de los cuales 104 eran federales y 107 estatales en 31 estados de la República; estos últimos son organismos descentralizados de los gobiernos de los estados. De los 211 institutos, 184 son tecnológicos industriales, 20 agropecuarios, seis del mar y uno forestal. Existen también seis centros especializados: Centro Nacional de Investigación y Desarrollo Tecnológico (CENIDET); Centro Interdisciplinario de Investigación en Docencia Técnica (CIDET), y cuatro son Centros Regionales de Optimización y Desarrollo de Equipo (CRODE) (Rubio, 2006).

${ }^{15}$ La institución se crea por decreto presidencial (publicado el 23 julio de 2014), como un órgano desconcentrado de la SEP, con "autonomía técnica, académica y de gestión" (Decreto de creación del TecNm, 2014: 1), constituido por 266 instituciones.
} 
tuciones de educación superior pública por medio de la calidad, la competencia y la productividad, la modificación estructural de los mercados de trabajo ante las exigencias del Tratado de Libre Comercio de América del Norte (TLC).

Desde una mirada local, cabe destacar que hasta inicios del año 2000 los institutos tecnológicos estaban agrupados en la Dirección General (DGEST) que dependían de la Secretaria de la SEP, en donde existía un modelo institucional organizacional y académico poco flexible y con una incipiente atención a las necesidades nacionales, regionales y locales.

Me interesa mostrar el archipiélago tecnológico del TecNM, cuya procedencia se ubica desde la creación de los primeros it en el país. Su crecimiento paulatino ha tenido momentos vertiginosos dependiendo de la política educativa construida en el país. A continuación se presenta la cobertura y los datos estadísticos del TecNM durante el ciclo escolar 2015-2016:

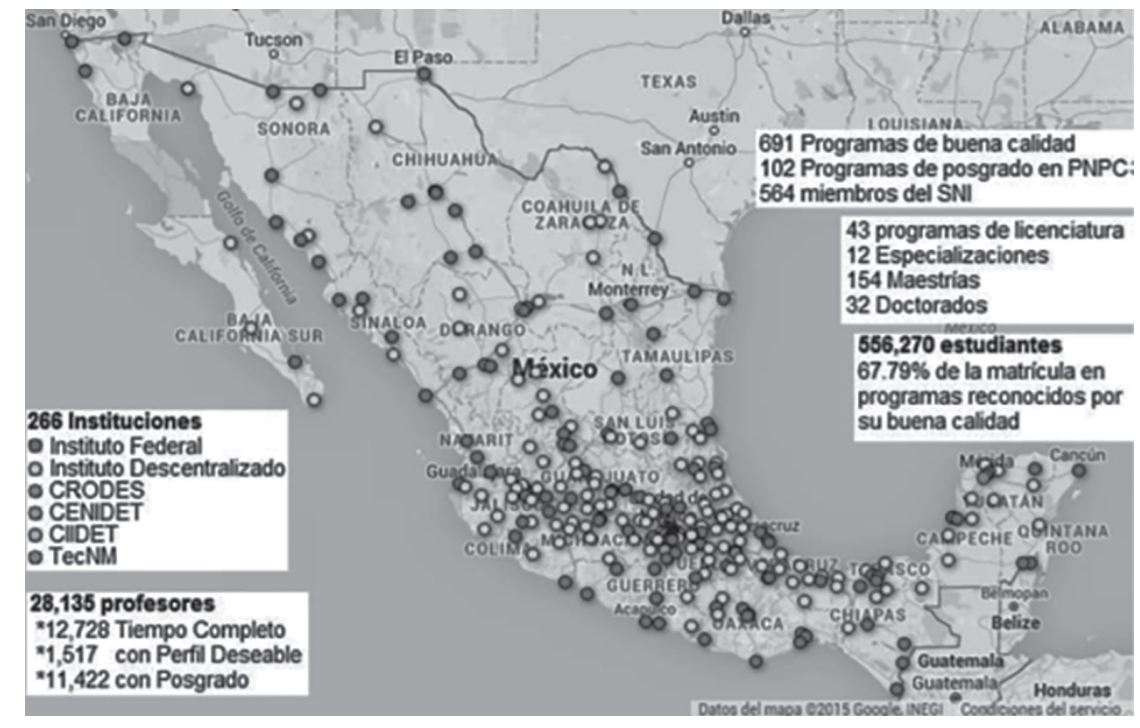

Fuente: Google INEGI (2015) y Gamino et. al. (2016

Buscar el reconocimiento del TecNM a nivel social, político y de recursos financieros, no ha sido fácil. Es por ello que el exdirector general del тесNM, Manuel Quintero Quintero, durante el foro El Tecnológico Nacional de México: Presente y futuro. Los institutos tecnológicos y su contribución a la ciencia, tecnología e innovación, ${ }^{16}$

${ }^{16}$ La finalidad del evento fue resaltar las grandes contribuciones que han realizado los institutos tecnológicos (Quintero, 2018). Participaron además del director del TecNM, el director general del Consejo Nacional de Ciencia y Tecnología (Conacyt), el presidente de la Comisión de Innovación del Consejo Coordinador Empresarial, Víctor Gutiérrez 
realizado en la LXIII Legislatura de la Cámara de Diputados en abril de 2018, señaló que

Es claro que alcanzar las grandes metas nacionales no será posible sin una educación de calidad y un desarrollo científico y tecnológico sustentable, que favorezca la generación de conocimiento y de una industria propia, y, por ende, una mayor independencia tecnológica y económica del país. A lo largo de 70 años los institutos tecnológicos han formado gran parte de los ingenieros que han sido actores fundamentales para el crecimiento y desarrollo del país en la industria petrolera, automotriz, energética, aeroespacial, agropecuaria, de alimentos, ambiental, y en el desarrollo de las tecnologías de la información, así como en la construcción de la infraestructura carretera, entre otros. (Quintero, 2018: 1). (El énfasis es mío).

Es importante destacar que aún en esta pugna por conseguir un mayor presupuesto aparecen términos cuya procedencia corresponde al programa cardenista (1934-1940) cuyo proyecto nacional era lograr la independencia tecnológica y económica del país, para favorecer una industria propia, pero que no coincide con el proyecto de país durante el gobierno de Enrique Peña Nieto, en donde hay huellas de los proyectos de Manuel Ávila Camacho (1940-1946) y Miguel Alemán Valdés (1946-1952). Aunque también emerge un nuevo lenguaje a partir del contexto actual: aeroespacial, energético, ambiental, tecnologías de la información, etc.

Actualmente el Tecnm se encuentra en una etapa de búsqueda y de consolidación, aún cuenta con múltiples retos. Uno de ellos, es el modelo educativo que pretende ser flexible y que aún se encuentra en proceso de implementación, por lo que la difusión y la recepción son relevantes. Considero que es necesario analizar los espacios de trabajo, capacitación y comunicación, que permitirá además examinar la apropiación y la resignificación por parte de los actores, así como evaluar si cubre o no con sus expectativas.

Estos actores del TecnM son clave ya que puedan hacer suyo o no el modelo académico, tal como está planteado, o bien transformarlo a partir de las formas culturales locales y las necesidades individuales y colectivas de las

Martínez, el presidente de la Red Nacional de Consejos y Organismos Estatales de Ciencia y Tecnología, José Alonso Huerta Cruz, el director de Innovación en la Secretaría de Economía, Salvador Orozco Fernández, y los diputados de los diferentes partidos políticos. Se firmó la Carta Declaratoria San Lázaro, cuyo objetivo es establecer el compromiso de la Cámara de Diputados para coadyuvar a que la institución logre sus metas en el desarrollo tecnológico, investigación e innovación (Boletín 5229 de la Cámara de Diputados (2018). 
comunidades académicas, estudiantiles o de gestión. Aquí es interesante el análisis de lo "instituido (lo fijo, lo estable) y lo instituyente (el cuestionamiento, la crítica y la propuesta opuesta o de transformación) que plantea Fernández (1994).

El principio filosófico del modelo sugiere un sujeto de aprendizaje y ciudadano del mundo. Para cumplir con las tareas de formación, principalmente de ingenieros, el TecNM reconoce que la etapa que inició con las máquinas de vapor (industria 1.0); la producción en cadena en donde se requería únicamente la energía eléctrica (industria 2.0); la automatización y el desarrollo de la electrónica (3.0) ya no son actuales. El ideal de plenitud está en la industria (4.0), considerada como de vanguardia (computación en la nube, realidad aumentada, ciberseguridad, sistemas de integración de manera horizontal y vertical, fabricación auditiva, big data, internet industrial) que estudiantes en ingeniería deben de aprender para atender las necesidades de la empresa.

Thomas Popkewitz ha evidenciado que "[...] las prácticas pedagógicas y las ciencias de la educación mantienen el sistema de razón que asume que la combinación correcta de políticas y ciencias proporcionará un mundo armonioso y estable frente a su condicionalidad" (2007: 433 y 434). El papel de la planeación y de toda reforma educativa pretende modificar las condiciones de las escuelas pero con la pretensión de cambiar también a la gente, como refiere el autor.

Desde esta perspectiva, sí que es un reto formativo para el тесNм, уa que su población estudiantil y docente no es homogénea. Habría que reflexionar sobre "el desfavorecido" o el rezagado, son población "peligrosa" para las aspiraciones no dichas y la normalización (Popkewitz, 2007) que se pretende a veces realizar. Por ejemplo, son evidentes los diferentes contextos sociales y culturales que vive actualmente el país, en donde el capital cultural de los actores clave del TecNM es distinto y en donde en algunos ITs o nominados actualmente como campus no cuentan con la infraestructura necesaria ni con internet. No es lo mismo ser estudiante de ingeniería del тесNм en el norte, que en el sur o centro de México, o bien de zonas urbanas, rurales o indígenas. Tampoco es lo mismo ser alumno de un iт federal que de un descentralizado.

La interrogante es ¿las políticas educativas toman en cuenta estas diferencias o más bien funcionan para borrar y reinscribir diferencias? Habría que considerar que los IT para muchos estudiantes es su esperanza de recibir educación superior, es su lugar de esperanza y el espacio para ser ingeniero, para perder el miedo de no "ser nadie", porque ahí simbolizan su estar en el mundo, en "ser alguien en la vida". Habría que investigar cuántos de ellos en su familia son los primeros que acceden a la educación superior. 
En términos generales, en este artículo se presentan algunas características que es necesario profundizar y articular. Es un reto para mí hacerlo en el momento coyuntural de transiciones que están ocurriendo en el país a partir del cambio de régimen con un proyecto si no totalmente diferente, sí con muchos matices que hay que analizar para comprender las políticas educativas y el ser ingeniero en el TecNM.

Ciertamente, el escenario político, ante la instauración del nuevo régimen de Andrés Manuel López Obrador, como presidente de México, se ha modificado. El 8 de enero de 2019 es nombrado el Dr. Enrique Fernández Fassancht, ${ }^{17}$ como nuevo director del TесNM. En su discurso de toma de posesión le otorga un reconocimiento al тесNM en relación con la matrícula, al afirmar que ésta es mayor que la suma a nivel superior de la UNAM, la UAM y el IPN (тесNм, 2019). Quiero destacar que aún después de 71 años de que se crearon los primeros ITs, las huellas del IPN, estos indicios de su comienzo no han desaparecido, aún siguen vigentes. La procedencia de los que han estado al frente en la toma de decisiones sigue siendo principalmente del IPN, así como muchos de sus profesores.

\section{A manera de conclusiones}

Se puede concluir que la política educativa no es determinante, pero sí crea condiciones para la identificación de los principales actores del TecNM. Las identidades se construyen en el ámbito de lo social, por lo que son históricas, institucionales y situadas. Permite reconocer las huellas de la procedencia y la emergencia, que están presentes en la actualidad, pero ha habido una resignificación de estas políticas por los actores que han construido las políticas educativas para intentar dar respuesta al contexto actual.

La tesis más importante que puedo sostener una vez revisados e interpretados algunos documentos de la política educativa en el тесNм, es que:

a) A la vez que se observan huellas de una procedencia negada (por los padres fundadores de los IT), la del IPN, al proclamar que no existían ante-

\footnotetext{
${ }^{17}$ El Dr. Fernández es doctor en Química (Fisicoquímica) de la Facultad de Química de la UNAM, maestro en la División de Ciencias Básicas e ingeniería de la uAM Unidad Iztapalapa y la licenciatura en la Escuela Superior de Ingeniería Química e Industrias Extractivas (ESIQUIE) del IPN. Fue el director del IPN hasta noviembre 2017; rector general de la UAM; coordinador de las Universidades Politécnicas de la SEP, y secretario general ejecutivo de la Asociación Nacional de Universidades e Instituciones de Educación Superior (ANUIES) (página Web del тесNм, 2019).
} 
cedentes en México, esta herencia no desaparece sino que se articula y combina con las diversas condiciones históricas de la propia trayectoria del archipiélago tecnológico.

b) Se mantiene en circulación y desplazamiento la concepción de ser ingeniero en los primeros tecnológicos fundadores (Siglo xx) cuya preocupación estaba centrada en la formación técnica para la solución de problemas de la región y del ámbito local. ${ }^{18}$ Por ejemplo, el primer ingeniero industrial abordó en su tesis el problema del agua del estado de Chihuahua. Pero también se modifica, durante el contexto de principios del siglo XXI, la fijación inicial de su sentido, por medio de las estrategias y acciones que se toman a través de la política educativa que se implementa, en la cual se impulsa la formación tecnológica para la formación de ingenieros que atiendan las necesidades de las empresas en cada región, muchas de las cuales no son nacionales sino que son extranjeras.

Me interesa también señalar algunas reflexiones como producto de la presente investigación; una de ellas es la importancia de las instituciones de educación superior públicas, como lo es el TecNM, en donde se construye un sentido de ser ingeniero en México y su relación con el proyecto de país que se esté impulsando.

Si bien esta es una mirada inicial, sí considero que hace falta: I) Investigar en relación con la configuración de los campos disciplinares "propios" y "no propios" que tienen relación con la formación de ingenieros; los problemas actuales exigen ir más allá de las barreras disciplinares. II) Realizar estudios sobre las prácticas educativas de formación de ingenieros que se desarrollan, para analizar los problemas que abordan, los conceptos, los métodos, las herramientas que utilizan para analizar los sistemas de razón (Popkewitz, 2007). III) Indagar las trayectorias de los docentes y de los estudiantes de la institución para conocer sus significaciones de ser ingeniero y los sentidos que construyen, pues forman parte de sus procesos subjetivos; esto permite dar cuenta de su existencia y el ser ingeniero en este mundo, nuestro país y la sociedad de los que forma parte. Iv) Evaluar el proyecto curricular vigente, en donde se analicen los planes y programas de estudio, para conocer su viabilidad con el actual proyecto de país.

El interés pedagógico es el de analizar las propuestas de formación que actualmente se desarrollan, de cómo se constituyen los sujetos educativos. Es

\footnotetext{
${ }^{18}$ El primer titulado de la carrera de ingeniería industrial fue Raúl Ambriz González, presentó su examen el 22 de diciembre de 1956 con la tesis "Análisis de abastecimiento de agua de la ciudad de Chihuahua". Es también considerado como el primero en toda la República Mexicana del naciente sistema nacional de Institutos Tecnológicos (Sáenz, 1999).
} 
necesario transgredir las prácticas educativas homogenizantes, echar mano de las diversas propuestas disciplinarias de vanguardia para construir un mundo mejor y de vida humana, así como de país, de comunidad. En esta tarea la formación de ingenieros es muy importante, si no queda anclado en una visión y un pensamiento de su profesión limitadas, centradas en certezas y no preparadas para la incertidumbre ante los vertiginosos cambios de la realidad.

\section{Referencias}

Buenfil, Rosa y Navarrete, Zaira (2018). Ernesto Laclau: Apropiaciones, debates y diseminación de su pensamiento en Latinoamérica. México, Plaza y Valdés/PAPDI.

Buenfil, Rosa (2011). Apuntes sobre los usos de la teoría en la investigación social. Consideraciones metodológicas en investigación social. España, Editorial Académica Española.

Buenfil, Rosa (2004). Argumentación y poder: La mística de la Revolución Mexicana rectificada, México, Plaza y Valdés-PAPDI.

Buenfil, Rosa (1994). Cardenismo. Argumentación y Antagonismo en Educación. México, DIE-Cinvestav-IPN/Conacyt.

Decreto de creación del Tecnológico Nacional de México (2014). Consultado el 17 de marzo de 2016, en http:/ / www.dof.gob.mx/nota_detalle.php?codigo=53 $53459 \&$ fecha $=23 / 07 / 2014$

Fernández, Lidia (1994). Instituciones educativas: dinámicas institucionales en situaciones críticas. Buenos Aires, Paidós.

Foucault, Michel (1992). Microfísica del poder. Madrid, La piqueta.

Foucault, Michel (2008). Tecnologías del yo y otros textos afines. Barcelona, Paidós. Traducción Mercedes Allendesalazar.

Galindo, Carlos (2010). Vuelta al Alma Mater. México, Instituto Tecnológico de Chihuahua.

Gamino, Arturo, Acosta, Mara y Pulido, Rocío (2016). “Modelo de formación dual del Tecnológico Nacional de México" en Revista de Investigación en Educación No. 14 (2), pp. 170-183. Consultado en http:/ / webs.uvigo.es/reined/

Guevara, Gilberto (comp.) (1985). La Educación Socialista en México. Antología, SEP-México, editorial El Caballito.

Partido Nacional Revolucionario (1934) La jira [sic] del General Lázaro Cárdenas. Síntesis ideológica México, Secretaría de prensa y propaganda del C. E. N. del P. N. R.

Popkewitz, T. (2007). "La razón de la esperanza cosmopolita como miedo a la diferencia" (trad. Rosa Nidia Buenfil Burgos) en Padierna, P. y Mariñez, R., Coords., Educación y Comunicación. Tejidos desde el Análisis Político de Discurso. México, Juan Pablos-PAPDI, pp. 433-466. 
Quintero, M. (2018) Informe de rendición de cuentas de conclusión de la Administración 2012-2018) en el тесNM. México, тесNM, consultado en enero 14 de 2019 en https://www.tecnm.mx/images/areas/INFORME_DE_RENDICION_DE_CUENTAS_DE_CONCLUSION_DELA_ADMINISTRACION_2012-2018.pdf

Rubio, Julio (2006) (Coordinador). La política educativa y la educación superior en México. 1996-2006: Un balance. México, FCE.

Ruiz, Estela (2008). “La profesionalización de la Ingeniería en México. Un análisis histórico desde las teorías de la profesión" en Fernández, Jorge (Coord). El mundo de las profesiones en el siglo XXI: perspectivas y enfoques. México, BUAPUAMEX, Fomento Editorial, pp. 209-254.

Ruiz, Mercedes (2001). Archipiélago educativo: espacios de formación del sujeto adulto, en Pensar lo educativo. Tejidos conceptuales (Gómez, M. y Orozco, B., Coordinadoras). México, Plaza y Valdés/Sade, pp. 75-88.

Sáenz, Ricardo (1999). “ .. y nació el primer Tecnológico del sistema de educación más grande de México" en 70 años testimonios de historia, presente y futuro de educación tecnológica (2018). México, тесNM/ iтch, pp. 13-42.

Weiss, Eduardo y Bernal, Enrique (2013). Un diálogo con la historia de la educación técnica mexicana en Perfiles Educativos. México, unAm-IISUE, Vol.xxxv, Núm. 139, pp. 151-170.

Tamez, Reyes (2006). Prólogo. En Rubio, J. (2006) (Coordinador). La política educativa y la educación superior en México. 1996-2006: Un balance. México, FCE, p. 12.

TecNM (2019). Matrícula 2012-2018. Consultada el 20 de mayo de 2019 en https:// sne.tecnm.mx/public/

TесNм (2019). El presidente López Obrador designa al doctor Fernández Fassnacht, Director General del тесNм. México, página Web, consultada el 14 de enero de 2019, en https:/ / www.tecnm.mx/tecnm/el-presidente-lopez-obradordesigna-al-doctor-fernandez-fassnacht-director-general-del-tecnm 
